\title{
Frühjahrstagung der Arbeitsgemeinschaft Knochentumoren 2018
}

\section{Tagungsbericht}

Die 90. Tagung der AG Knochentumoren fand vom 4. bis 5. Mai in Leipzig in den Räumen des Instituts für Pathologie sowie im Zentralen Forschungsgebäude des Universitätsklinikums Leipzig statt. Priv.-Doz. Dr. Torsten Prietzel (jetzt Klinikum Blankenhain, vormals Klinik für Orthopädie und Unfallchirurgie des Univ. Klinikums Leipzig) als Gastgeber und sein Team sorgten als lokale Veranstalter dafür, dass das Arbeitstreffen reibungslos und in äußerst angenehmer Atmosphäre ablief. Alle Tagungsteilnehmer konnten zwei wissenschaftlich interessante Tage mit engagierten Diskussionen erleben. Für diese optimalen Bedingungen wissen sich die über 60 Tagungsteilnehmer den Gastgebern zu grossem Dank verpflichtet.

Die Tagung begann mit der Vorstandssitzung (15.00 Uhr bis $16.00 \mathrm{Uhr}$ ) und wurde mit der Präsentation von ad-hoc Fällen fortgesetzt (16.00 Uhr bis 18.00 Uhr). Insgesamt wurden 4 Fälle vorgestellt und diskutiert. Diese Fälle, die kurzfristig über das Sekretariat der AGKT (sekretariat@agkt. org) angemeldet oder spontan eingebracht wurden, beinhalten aktuelle diagnostische oder therapeutische Probleme, zu denen Expertenmeinungen aus dem Kreis der AGKT erbeten sind. Darunter waren ein Fall mit aggressiver Radiologie, aber eher reaktiver, nicht-tumoröser Histologie, der noch weiter geklärt und dann im Herbst erneut vorgestellt werden soll, sowie ein zunächst als gutartig eingeschätzter Knorpeltumor im prox. Femur mit mehr als 10-jährigem Verlauf und mehreren Rezidiven, der aktuell in ein (low-grade) Chondrosarkom übergegangen war. Ein weiterer Fall betraf eine knochenbildende Kieferläsion, deren Klärung jedoch noch nicht abgeschlossen ist und bei der ein Osteoblastom oder ein Osteosarkom diskutiert wurden. Der letzte Fall betraf ein 11-jähriges Mädchen mit einer xanthogranulomatösen Osteomyeltis der Tibia.

Daran anschließend fand das wissenschaftliche Programm (18.00 Uhr bis 19.00
Uhr) mit folgenden Vorträgen statt, welche im Anschluss diskutiert wurden:

- T. Prietzel ${ }^{1}$, Richter A. ${ }^{2}$, D. Zajonz ${ }^{2}$, M. Schmidt ${ }^{3}$, S. Schleifenbaum ${ }^{3}$, N. Hammer $^{4}$, R. Grunert ${ }^{5}$. Weichteilrekonstruktion und Komplikationsprophylaxe in der Tumorendoprothetik. ${ }^{1}$ Department of Orthopaedic and Trauma Surgery, HELIOS Clinic Blankenhain, Wirthstraße 5, 99444 Blankenhain, Germany; ${ }^{2}$ Department of Orthopaedic, Trauma and Plastic Surgery, University of Leipzig, Liebigstraße 20, 04103 Leipzig, Germany; ${ }^{3}$ Department of Orthopaedic, Trauma and Plastic Surgery, ZESBO, University of Leipzig, Semmelweisstraße 14, 04103 Leipzig, Germany; ${ }^{4}$ Department of Anatomy, University of Otago, 270 Great King St., Dunedin 9016, New Zealand; ${ }^{5}$ Fraunhofer Institute for Machine Tools and Forming Technology, Fraunhofer IWU, Nöthnitzer Straße 44, 01187 Dresden, Germany

- S. Hesse. Einsatzmöglichkeiten der Positronen-Emissions-Tomographie (PET) bei primären Knochentumoren. Klinik und Poliklinik für Nuklearmedizin der Universität Leipzig)

Kurzfassungen aller Vorträge sind im Anschluss an den Tagungsbericht zu finden. (S. $181 \mathrm{ff}$ ).

Anschließend ging es vom Universitätsklinikum via U-Bahn und einem kurzen Fußmarsch vom Marktplatz zum neuen Unihauptgebäude am Augustusplatz und dem Panoramatower, von dem aus sich den Teilnehmern bei strahlendem Wetter ein imposanter Blick über Leipzig bot.

\section{Mitgliederversammlung}

Der Samstag begann mit der nichtöffentlichen Mitgliederversammlung (8.30 Uhr bis 9.30 Uhr). Nach Abhandlung der Tagesordnung wurde beschlossen, Frau Dr. Anna Hirschmann, Leiterin der Abteilung für Muskuloskelettale Radiologie am Universitätsspital Basel, und Frau Dr. Carolin
Knebel, Oberärztin der Sektion Tumororthopädie am Klinikum rechts der Isar der TU München, als neue Mitglieder in die AGKT aufzunehmen.

\section{Wissenschaftliche Falldiskussion}

Als Hauptteil des Samstagvormittags folgte dann ab 9.30 Uhr die wissenschaftliche Falldiskussion vorher eingereichter Fälle, deren klinische Informationen, Radiologie und eingescannten Schnittpräparate den Mitgliedern und Gästen vorab im Internet zur Einsichtnahme und Beurteilung zur Verfügung standen (Moderation: Prof. Dr. Elisabeth Bruder, Basel).

Insgesamt wurden 16 Fälle diskutiert. Das Altersspektrum reichte von 10 bis 78 Jahren. Betroffen waren die langen Röhrenknochen $(9 \mathrm{x})$, die Skapula, der Fuß (je $3 \mathrm{x})$ sowie die Wirbelsäule und der Unterkiefer. Eine Läsion (Riesenzelltumor) trat multifokal auf. 6 Fälle waren gutartige Läsionen mit unterschiedlichen klinisch-diagnostischen (Osteomyelitis) oder morphologischen Besonderheiten (je ein Fall eines fibrocartilaginäres Mesenchymomsohne GNAS1-Mutation- und einer fibrocartilaginären fibrösen Dysplasie - mit GNAS1-Mutation) sowie ein Skapulatumor mit der morphologisch nicht entscheidbaren Differentialdiagnose symplastische/pseudoanaplastische aneurysmatische Knochenzyste vs. teleangiektatisches Osteosarkom. Ein Fall zeigte den Übergang eines gutartigen Knorpeltumors (Enchondroms) nach jahrelangem Verlauf in ein Chondrosarkom, neun Fälle betrafen (teils niedrig) malige Tumoren (ein nicht weiter klassifizierbares Hämangioendotheliom, zwei Chondrosarkome, davon ein Fall bei M.Ollier, ein extraskeletales myxoides Chondrosarkom mit Übergreifen auf die Metatarsalia, zwei high-grade Osteosarkome, ein Myoepitheliom des Knochens, eine Non-Langerhanszell-Histiozytose/fragl.

Erdheim-Chester-Erkrankung sowie eine Karzinommetastase, die sich radiologisch wie ein primärer Knochentumor im distalen Femur manifestierte).

Für die Darstellung und Diskussion Ihres Falles 1085 einer fibrocartilaginären fibrösen Dysplasie wurden Frau Dr. Maya 
Niethard, Priv.-Doz. Dr. Per-Ulf Tunn, und Priv.-Doz. Dr. Mathias Werner aus Berlin mit dem Preis für die beste Fallpräsentation ausgezeichnet.

Die Tagung wurde gegen 14.00 Uhr mit einem nochmaligen Dank an das Leipziger Team für die gute Organisation der Veranstaltung und die hervorragende Betreuung beendet.

\section{Zukünftige Tagungen}

Die Herbsttagung wird wie üblich in München (19. und 20. Oktober 2018) stattfinden. Vorangehen wird wieder am Freitagvormittag eine 4-stündige Fortbildungsveranstaltung, die gemeinsam von der Deutschen Gesellschaft für muskuloskelettale Radiologie (DGMSR) und der AGKT zum Thema: „Knochentumoren: Pathologie und Radiologie" organisiert wird. Weitere Einzelheiten können der Homepage (www. agkt.org) entnommen werden. Die Frühjahrstagung 2019 wird auf Einladung von Prof. Sven Seiwerth anfangs Mai in Zagreb stattfinden.

Falls Interesse besteht, als Gast an den Tagungen der $A G$ Knochentumoren teilzunehmen, können weitere Informationen über das Sekretariat der AGKT in Basel abgerufen werden (sekretariat@agkt.org).

\section{Kurzfassung der Vorträge}

\section{Weichteilrekonstruktion und Komplikationsprophylaxe in der Tumorendoprothetik \\ Hintergrund}

Die weite Tumorresektion nach Enneking stellt in der Mehrzahl der Fälle die adäquate operative Therapie im Rahmen der kurative Behandlung primärer maligner Knochentumoren dar. Die weitreichenden Rekonstruktionsmöglichkeiten der modernen Tumorendoprothetik erlauben dennoch in der Mehrzahl der Fälle ein extremitätenerhaltendes Vorgehen. Auch bei palliativem Therapieansatz ist auf Grund der gehäuften gelenknahen Tumorlokalisation in vielen Fällen eine endoprothetische Versorgung die bevorzugte Option. Mit ca. 50\% Anteil an der Tumorendoprothetik kommt dabei dem proximalen Femurersatz die größte Bedeutung zu. Modulare Systeme haben sich durchgesetzt, da diese eine in- traoperative Implantatanpassung und somit dem Operateur einen großen Handlungsspielraum ermöglichen, weshalb die Wiederherstellung der knöchernen Kontinuität kaum noch problematisch ist. Die meisten Komplikationen resultieren aus Problemen im Zusammenhang mit der Rekonstruktion der Weichteile. Deren mangelhafte dauerhafte Anbindung an das metallische Megaimplantat führt nicht selten zu Komplikationen, wie beispielsweise $\mathrm{zu}$ periprothetischen Seromen mit resultierender Wundsekretion, zu Wundheilungsstörungen und Infektionen, zur mangelhaften Muskelanbindung bzw. Muskelinsuffizienz und zur Luxation. Zur Verminderung der genannten Risiken nutzen wir seit Jahren regelhaft einen Trevira-Anbindungsschlauch ( Abb. 1). Zur Luxationsprophylaxe rekonstruieren wir außerdem in fast allen Fällen die Hüftgelenkkapsel und setzen bevorzugt Duoköpfe ein.

Im Rahmen dieser Studie sollte der Einfluss von Kapselrekonstruktion, Duokopfeinsatz und Nutzung des Trevira-Anbindungsschlauchs auf die Luxations- und Revisionsrate nach proximalem Femurersatz vergleichend analysiert werden.

\section{Patienten/Methoden}

Die im Zeitraum zwischen 10/2005 und 4/2015 in der Klinik für Orthopädie, Unfallchirurgie und Plastische Chirurgie des Universitätsklinikums Leipzig (bis 2014 Orthopädische Klinik und Poliklinik) unter Verwendung eines modularen Tumorendoprothesensystems (MML-System der Fa. AQ Implants, vorher Fa. ESKA-Implants bzw. Fa. Orthodynamics) mit proxi- malem Femurersatz versorgten Patienten wurden retrospektiv analysiert.

Patienten mit Rekonstruktion der Gelenkkapsel wurden der Studiengruppe (SG) zugeordnet, jene mit Kapselresektion der Kontrollgruppe (KG).

Sämtliche verfügbaren Daten wurden ausgewertet (elektronisches Krankenblatt, OP-Dokumentation, Kodierungsdaten und radiologische Befunde im PACS-System). Der Fokus der Nachuntersuchung lag auf der Ermittlung von Luxationsereignissen, notwendigen Revisionsoperationen und sonstigen Komplikationen. Bei Nichterreichbarkeit oder verstorbenen Patienten erfolgte die Befragung naher Angehöriger oder des Hausarztes, ob gravierende Komplikationen wie beispielsweise Luxationen eingetreten oder erneute Operationen notwendig waren.

\section{Ergebnisse}

Im Untersuchungszeitraum wurden $91 \mathrm{~Pa}$ tienten mit einem proximalen Femurersatz (pFE) versorgt, davon 41 Fälle mit Rekonstruktion und 50 Fälle mit Resektion der Gelenkkapsel. Das mittlere Follow-Up betrug 14,3 Monate in der SG und 24 Monate in der KG. Im gesamten Kollektiv erfolgte der Eingriff 49 mal auf Grund von Komplikationen nach Hüftendoprothetik und 42 mal wegen eines Tumors, davon $11 \mathrm{Mal}$ in kurativer Intention und $31 \mathrm{mal}$ als Palliativeingriff. In der SG wurden ein von fünf Primärtumorpatienten mit einer Totalendoprothese (TEP) und die übrigen vier von fünf mit Duokopf (DK) versorgt. Bei allen 21 palliativen Fällen in der SG kam ein DK zum Einsatz. Insgesamt erfolgte in der SG
Abb. 1

Proximaler Femurersatz (Totalendoprothese) links mit TreviraAnbindungsschlauch in situ - unmittelbar vor Durchführung der Kapselnaht und Muskelfixierung

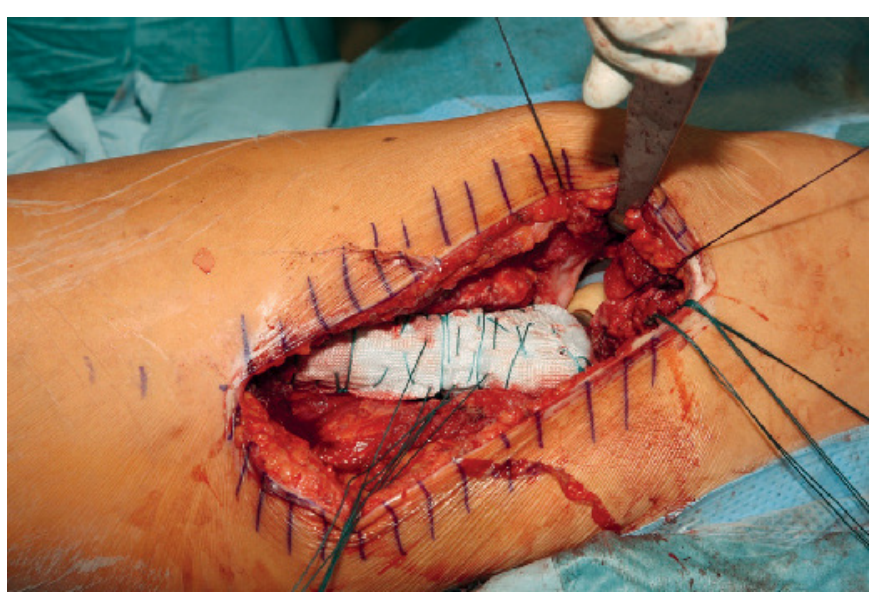


bei 11 Patienten eine TEP- und bei $30 \mathrm{~Pa}$ tienten eine DK-Versorgung. Sechs von 41 Patienten erlitten eine Luxation und bei 10 von 41 Patienten war eine operative Revision notwendig. Fünf von sechs Luxationen ereigneten sich nach TEP- und nur eine von sechs Luxationen nach DK-Implantation. Somit kam es in der SG bei einem von 30 Duoköpfen zur Luxation (Luxationsrate 3,3\%), während bei 7 von 30 eine operative Revision notwendig war. Im Gegensatz dazu luxierten fünf von 11 TEPs, während die Revisionsrate drei von 11 betrug.

In der KG wurden 34 von 50 Patienten im Rahmen von HTEP-Revisionen und 16 von 50 Patienten auf Grund eines Tumorleidens versorgt. Insgesamt waren in der KG 17 Patienten von Luxationen betroffen (34\%).

\section{Diskussion}

Die Verfügbarkeit modularer Tumorendoprothesensysteme sichert dem Operateur einen erheblichen intraoperativen Handlungsspielraum und ermöglicht in der Mehrzahl der Fälle eine extremitätenerhaltende operative Therapie. Gleichzeitig geht diese sogenannte Megaendoprothetik mit deutlich erhöhten Risiken einher, deren Reduktion bzw. Beherrschung angestrebt werden muss. Die Luxation ist neben der Infektion beim proximalen Femurersatz teratur werden beispielsweise Luxationsraten von $1,7 \%(1), 2 \%(3), 14,3 \%(6), 14,8 \%$ (7), 37\% (5) und $42 \%$ (7) genannt. Dem stehen Infektionsraten von $1,9 \%$ (2) bis $33 \%$ (8) gegenüber. Weitere Komplikationsmöglichkeiten sind Endoprothesen-Lockerungen, -Diskonnektionen oder -Brüche. Im eigenen Patientengut hat sich zur Luxationsprophylaxe die kombinierte Anwendung von Kapselrekonstruktion und Duokopf bewährt. In den Fällen, in denen beides möglich war, fanden wir mit 3,3\% eine Luxationsrate, die im Bereich der primären Hüftendoprothetik lag. Im Vergleich zur Kontrollgruppe mit einer Luxationsrate von $34 \%$ konnte damit das Luxationsrisiko um mehr als $90 \%$ reduziert werden. Erhöhte Revisionsraten oder andere Nachteile infolge der Kapselrekonstruktion wurden nicht beobachtet. Im Rahmen der Weichteilrekonstruktion nach Resektion des koxalen Femur und proximalem Fedie bedeutendste Komplikation. In der Li-

murersatz setzen wir routinemäßig einen Trevira-Anbindungsschlauch ein, um die intraoperative Muskelanbindung durch Nähte und eine dauerhafte narbige Einheilung im weiteren Verlauf zu ermöglichen (4). Im Rahmen der Muskelrekonstruktion werden die pelvitrochantäre Muskulatur, der Musculus iliopsoas und der Musculus quadriceps femoris sorgfältig unter Nutzung des Anbindungsschlauchs am Implantat fixiert. Um Vor- oder Nachteile dieser operativen Technik bewerten zu können, sind jedoch weitere Untersuchungen unter Einschluss größerer Fallzahlen erforderlich. Zur weiteren Optimierung der Weichteilanbindung soll ein Forschungsprojekt beitragen, welches im Rahmen des Netzwerkes Endoprothetik („Kunstgelenk“, https://www.kunstgelenk.eu) vorangetrieben wird.

\section{Zusammenfassung}

Die Schonung und Rekonstruktion der Hüftgelenkkapsel in Kombination mit dem Einsatz eines Duokopfs führte im untersuchten Patientengut zu einer um mehr als $90 \%$ reduzierten Luxationsrate, weshalb dieses Vorgehen empfohlen werden kann, vorausgesetzt es ist im Einzelfall operationstechnisch realisierbar. Der Einsatz des Trevira-Anbindungsschlauches scheint die weichteil-assoziierten Komplikationen zu vermindern. Diesbezüglich sind jedoch weitere Untersuchungen erforderlich.

Torsten Prietzel ${ }^{1}$, Andy Richter ${ }^{2}$, Dirk Zajonz²,

Michael Schmidt ${ }^{3}$, Stefan Schleifenbaum ${ }^{3}$, Niels Hammer ${ }^{4}$, Ronny Grunert ${ }^{5}$

${ }^{1}$ Abteilung Orthopädie und Unfallchirurgie, Helios Klinik Blankenhain, Blankenhain,

Deutschland

${ }^{2}$ Klinik für Orthopädie, Unfallchirurgie und Plastische Chirurgie, Universitätsklinikum Leipzig, Leipzig, Deutschland

${ }^{3}$ Klinik für Orthopädie, Unfallchirurgie und Plastische Chirurgie, Universitätsklinikum Leipzig, Leipzig, Deutschland

${ }^{4}$ Department of Anatomy, University of Otago, Dunedin, New Zealand

${ }^{5}$ Fraunhofer-Institut für Werkzeugmaschinen und Umformtechnik, Institutsteil Dresden,

Dresden, Deutschland

\section{Literatur}

1. Bickels J, Meller I, Henshaw RM, Malawer MM. Reconstruction of hip stability after proximal and total femur resections. Clinical Orthopaedics and Related Research 2000; 375: 218-230.

2. Capanna R, Scoccianti G, Frenos F, Vilardi A, Beltrami G, Campanacci DA. What was the survival of megaprostheses in lower limb reconstructions after tumor resections? Clinical Orthopaedics and Related Research 2015; 473(3): 820-830.

3. Gebert C, Wessling M, Gotze C, Gosheger G, Hardes J. The Modular Universal Tumour And Revision System (MUTARS(R)) in endoprosthetic revision surgery. International Orthopaedics 2010; 34(8): 1261-1265.

4. Gosheger G, Hillmann A, Lindner N, et al. Soft tissue reconstruction of megaprostheses using a trevira tube. Clinical Orthopaedics and Related Research 2001; 393: 264-271.

5. Haentjens P, Boeck H de, Opdecam P. Proximal femoral replacement prosthesis for salvage of failed hip arthroplasty: Complications in a 2-11 year follow-up study in 19 elderly patients. Acta Orthopaedica Scandinavica 1996; 67(1): 37-42.

6. Hardes J, Budny T, Hauschild G, et al. Der proximale Femurersatz in der Revisionsalloarthroplastik. Z Orthop Unfall 2009; 147(06): 694-9.

7. Salis-Soglio G von, Ghanem M, Meinecke I, Ellenrieder M, Klinger H, Kirchhoff C. Modulares Endoprothesensystem München-Lübeck (MML). Orthopäde 2010; 39(10): 960-967.

8. Shih S. Proximal femoral megaprosthesis for failed total hip arthroplasty. Chang Gung medical journal 2007; 30(1): 73-80.

\section{Einsatz der PET bei primären Knochentumoren}

Die nicht-invasive Diagnostik mittels Fluor-18 markierter Fluordesoxyglukose (FDG) und der Positronen-Emissions-Tomographie (PET) ist in der Onkologie weit verbreitet. Das Verfahren beruht auf einer vermehrten Expression von GlukoseTransportern (GLUT1) beziehungsweise einer vermehrten Hexokinase-Aktivität in Tumoren, insbesondere in denen mit hoher Proliferation. Entsprechend wird vermehrt FDG phosphoryliert und in der (Tumor-)Zelle akkumuliert, sodass es bildgebend mit der PET in Kombination mit der Computer-Tomographie (CT) als PET-CT erfasst und semi-quantitativ anhand eines standardisierten Uptake-Werts (SUV) dargestellt werden kann. Die meisten malignen primären Knochentumore wie das Osteosarkom und das Ewing-Sarkom weisen eine hohe $\left[{ }^{18} \mathrm{~F}\right]$ FDG-Avidität auf; andererseits können jedoch niedrig-gradige Chondrosarkome eine nur geringe $\left[{ }^{18} \mathrm{~F}\right]$ FDG-Mehraufnahme zeigen und be- 